\title{
Correlation between Magnetic Resonance Imaging Characteristics of the Patellar Tendon and Clinical Scores in Osgood-Schlatter Disease
}

\author{
Dhong Won Lee, $\mathrm{MD}^{1}$, Min Jeong Kim, $\mathrm{MD}^{2}$, Woo Jong Kim, $\mathrm{MD}^{3}$, Jeong Ku Ha, $\mathrm{MD}^{4}$, and Jin Goo Kim, $\mathrm{MD}^{5}$ \\ ${ }^{1}$ Department of Orthopedic Surgery, Daejeon Military Hospital, Daejeon; ${ }^{2}$ Department of Raiology, Ewha Womans University Mokdong Hospital, Seoul; ${ }^{3}$ Department \\ of Orthopedic Surgery, Soonchunhyang University Cheonan Hospital, Cheonan; ${ }^{4}$ Department of Orthopedic Surgery, Inje University Seoul Paik Hospital, Seoul; \\ ${ }^{5}$ Department of Orthopedic Surgery, Konkuk University Medical Center, Seoul, Korea
}

Purpose: This study aims to evaluate magnetic resonance imaging (MRI) findings in young adults with symptomatic Osgood-Schlatter disease (OSD) and compare those in young adults without OSD.

Materials and Methods: We compared MRI findings between young adults with OSD (OS group, $\mathrm{n}=30$ ) and the equivalent number of young adults without OSD (control group). Visual analog scale scores and Kujala scores were evaluated and correlation analysis was performed in the OS group. Results: In the OS group, MRI revealed that the patellar tendon was attached to the tibia more widely, resulting in a reduced free tendon portion, and more proximally to the articular surface $(\mathrm{p}<0.001)$. The correlation analysis between MRI findings and clinical scores showed statistically significant correlations $(\mathrm{p}<0.01)$. In the OS group, $43 \%$ presented with patellar tendinopathy or bone marrow edema at the distal attachments.

Conclusions: Compared to the control group, the relatively small free portion and relatively proximal attachment of the patellar tendon were observed with MRI in the OS group. The free portion of the patellar tendon was positively correlated with the clinical scores. Patellar tendinopathy was also frequently encountered in the OS group.

Keywords: Knee, Tibia, Patellar tendon, Osgood-Schlatter disease, Tendinopathy, Magnetic resonance imaging

\section{Introduction}

Osgood-Schlatter disease (OSD) has been known as a disease that causes traction apophysitis or stimulatory symptoms due to traction of the patellar tendon acting on the eminence of the tibial tuberosity during childhood and adolescence in which vigorous growth takes place ${ }^{1)}$. Despite that favorable natural progress has been recognized as a self-limiting disease in $90 \%$ of

Received July 13, 2015; Revised September 13, 2015;

Accepted September 18, 2015

Correspondence to: Jin Goo Kim, MD

Department of Orthopedic Surgery, Konkuk University Medical Center, 120 Neungdong-ro, Gwangjin-gu, Seoul 05030, Korea

Tel: +82-2-2270-0028, Fax: +82-2-2270-0023

E-mail: boram107@hanmail.net

This is an Open Access article distributed under the terms of the Creative Commons Attribution Non-Commercial License (http://creativecommons.org/licenses/by-nc/4.0/) which permits unrestricted non-commercial use, distribution, and reproduction in any medium, provided the original work is properly cited. the patients, severe pain in the tibial tuberosity and difficulties in daily life are often complained by athletes or military personnel who frequently perform activities that cause stress on the knee joint regardless of the completion of the growth ${ }^{2)}$. Various treatment methods including surgical treatment have been performed for the patients with severe symptoms refractory to conservative treatment $^{2-8)}$; however, cases where the symptoms persist even after surgical treatment have often been reported ${ }^{3,4,9-12)}$. This has been thought to be due to the lack of research on pathophysiology and clinical progress of the disease.

The biomechanics and pathophysiology of the proximal tibial tuberosity are important for young adults beyond the growth period because in cases where the osseous fragments (ossicles) remain unhealed, the symptoms are caused by the moment arm of the force that is different from the normal individuals due to the attachment of the patellar tendon in the proximal tibial tuberosity $^{13)}$. In order to determine how OSD that developed during the growth period triggers the symptoms beyond the growth period, radiological evaluation in adulthood is required and this can also 
be used to predict the progress of the disease after treatment. Few studies have analyzed OSD in young adults beyond the growth period using radiological evaluation. In addition, to our knowledge, the relationship between specific radiological findings and clinical assessments of the disease after the growth period has never been addressed in studies. Hence, we conducted a study using magnetic resonance imaging (MRI) that allows analysis of the bones and soft tissues upon the completion of growth. The purpose of this study was to investigate the radiological characteristics of OSD using MRI and assess their relations with clinical scores in young adults to determine the clinical relevance of MRI and help in surgery planning.

In this study, we analyzed MRI findings of the knee in young adult patients with symptomatic OSD beyond the completion of growth and compared with those in young adults without OSD. The authors also analyzed the correlation between the MRI findings and clinical scores in the OSD patients. Our hypothesis was 1) that the two groups would show a difference in the patellar tendon attachment, 2) clinical scores would be affected by specific MRI findings, and 3) patellar tendinopathy would be common in the OSD patients.

\section{Materials and Methods}

\section{Subjects}

A retrospective observational study was conducted on young adult male patients who visited the outpatient clinic at our hospital between May 2013 and March 2015; suffered from major clinical symptoms of OSD including pain and tenderness in the tibial tuberosity and limitation in squatting for more than 1 year continuously; presented with ossicles on the lateral view of the knee joint; and had an MRI examination. A total of 30 patients with OSD were included in the study excluding those with open physeal plate in the knee joint or with a clear trauma in the injured region. Among the patients who visited the clinic during the same period due to knee joint disease, 30 young adults without OSD were selected as control group by matching age, gender, and height to those of the patients with OSD (OS group) (Table 1). Visual analog scale (VAS) scores and Kujala scores were evaluated in the OS group at the time of visit. The study protocol was approved by the Institutional Review Board of Armed Forces Daejeon Hospital.

\section{Radiological Evaluation}

In simple radiography, the degree of patella alta/infera was evaluated by measuring the Insall-Salvati ratio on the lateral view of the knee joint ${ }^{14)}$. The length of the patellar tendon in the OS group was measured by referring to the tibial tuberosity as in the control group due to the shortening of the patellar tendon caused by ossicles. By using the image of 1.5T MRI (Intera Achieva; Philips, Amsterdam, The Netherlands), the proportion of the attachment in the region between the most proximal region of the tibial tuberosity or the most superior portion of the ossicles and the most distal portion of the tibial tuberosity to the total length of the patellar tendon was measured; the proportion of the region from the most proximal region of the tibial tuberosity or the most superior portion of the ossicles to the most distal portion of the tibial tuberosity in the total length of the region from the tibial articular surface to the most distal portion of the tibial tuberosity was measured ${ }^{13)}$; and the thickness of the patellar tendon and intensity of the signal were identified in order to determine the presence of comorbidity, such as patellar tendinopathy ${ }^{13,15)}$. On T1-weigted sagittal images, the free patellar tendon proportion [FPTP à $=(1) /(2)]$ was calculated by defining (1) as the distance between the inferior patella and the most proximal attachment site of the patellar tendon onto the tibial tuberosity or the ossicle and (2) as the distance between the inferior patella and the most distal attachment site of the patellar tendon onto the tibial tuberosity $^{13)}$. The tibial attachment proportion [TAP à=(3)/(4)] was calculated defining (3) as the distance between the most proximal attachment of the patellar tendon onto the tibial tuberosity or the ossicle and the most distal region of the tibial tuberosity and (4) as the distance between the tibial articular surface and the most distal region of the tibial tuberosity (Fig. 1$)^{13)}$. The presence of patellar tendinopathy was examined 1) by measuring the thickness of the attachment sites in the tibial tuberosity or in the ossicle, and 2) by evaluating the findings of peritendinous fluid or edema, substantial tendinopathy, bone marrow edema, and partial tear on T2-weighted sagittal images ${ }^{15,16}$.

Table 1. Patient Demographics

\begin{tabular}{lccc}
\hline \multicolumn{1}{c}{ Characteristic } & $\begin{array}{c}\text { OS group } \\
(\mathrm{n}=30)\end{array}$ & $\begin{array}{c}\text { Control } \\
\text { group }(\mathrm{n}=30)\end{array}$ & p-value \\
\hline Age $(\mathrm{yr})$ & $21.8 \pm 1.6$ & $21.6 \pm 1.8$ & 0.767 \\
Sex $($ male $)$ & & & 1.000 \\
Height $(\mathrm{cm})$ & $171.2 \pm 5.0$ & $170.5 \pm 4.8$ & 0.605 \\
Body mass index $\left(\mathrm{kg} / \mathrm{m}^{2}\right)$ & $22.9 \pm 1.2$ & $22.7 \pm 1.1$ & 0.599 \\
Duration of symptom $(\mathrm{mo})^{\mathrm{a})}$ & $15.2 \pm 2.4$ & & \\
\hline
\end{tabular}

Values are presented as mean \pm standard deviation.

OS: Osgood-Schlatter disease.

a) The values were evaluated only in the OS group. 
A

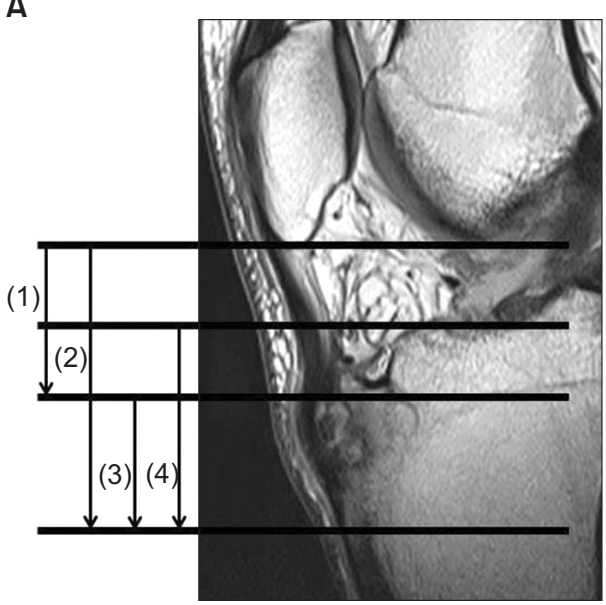

B

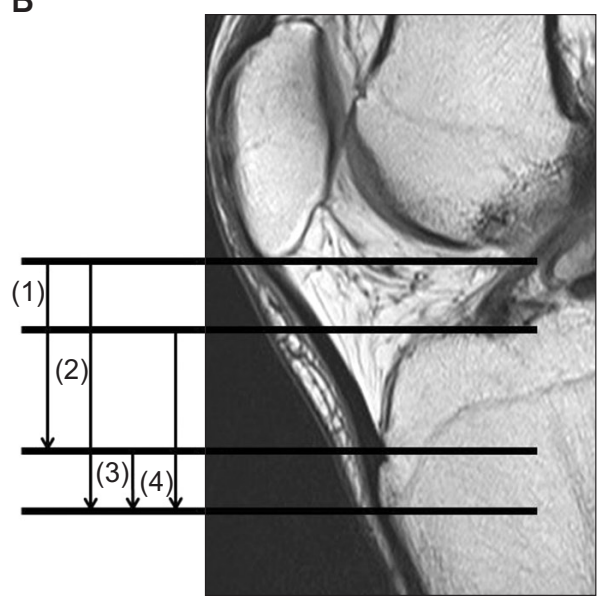

Fig. 1. Magnetic resonance imaging scans of a patient with Osgood-Schlatter disease (A) and a patient in the control group (B). (1): the distance between the inferior patella and the most proximal attachment site of the patellar tendon onto the tibial tuberosity or the ossicle, (2): the distance between the inferior patella and the most distal attachment site of the patellar tendon onto the tibial tuberosity, (3): the distance between the most proximal attachment of the patellar tendon onto the tibial tuberosity or the ossicle and the most distal region of the tibial tuberosity, (4): the distance between the tibial articular surface and the most distal region of the tibial tuberosity.

\section{Statistical Analysis}

SPSS ver. 17.0 (SPSS Inc., Chicago, IL, USA) was used for all statistical analysis. For analysis of the radiographic values, independent $t$-test was conducted. The level of statistical significance was determined as 0.05 . Radiological measurements were performed by one surgeon (Lee) from the department of orthopedic surgery and one physician (Kim MJ) from the department of radiology each with an interval of two weeks. Intraclass correlation coefficients (ICCs) were calculated to determine intra-observer and inter-observer reliability of the MRI measurements. The Pearson's or Spearman's correlation coefficient was used to characterize the relationship between FPTP à and clinical scores and between TAP à and clinical scores in the OS group.

\section{Results}

The location of the patella evaluated by the Insall-Salvati method was within the normal range in both groups, showing no statistically significant intergroup difference $(\mathrm{p}=0.817)$. The average ratio of the distance between the inferior patella and the most proximal attachment site of the patellar tendon onto the tibial tuberosity or the ossicle to the distance between the inferior patella and the most distal attachment site of the patellar tendon was $0.55 \pm 0.09$ in the OS group and $0.77 \pm 0.11$ in the control group; hence a statistically significant intergroup difference was observed $(\mathrm{p}<0.001)$. This result indicates that the area of tibial attachment of the patellar tendon compared to the total length of the patellar tendon was wider in the OS group. In other words, the patellar tendon of the OS group has less portion of free tendon; hence the length of the patellar tendon that is not attached onto the tibia is relatively short. The average ratio of the distance between the most proximal attachment of the patellar tendon onto the tibial tuberosity or the ossicle and the most distal region of the tibial tuberosity to the distance between the tibial articular surface and the most distal region of the tibial tuberosity was $0.67 \pm 0.08$ in the OS group and $0.29 \pm 0.07$ in the control group; hence a statistically significant intergroup difference was observed $(p<0.001)$. This result means that the tibial attachment of the patellar tendon is more proximal in the OS group. There was a statistically significant difference in the average thickness of the patellar tendon in the attachment site at the inferior patella as it was measured as $3.7 \pm 0.9 \mathrm{~mm}$ and $2.2 \pm 0.3 \mathrm{~mm}$ in the OS group and control group, respectively $(\mathrm{p}<0.001)$. The average thickness of the patellar tendon in the attachment site in the tibial tuberosity or the ossicle showed statistically significant intergroup difference as it was measured as $7.2 \pm 0.7 \mathrm{~mm}$ in the OS group and $4.0 \pm 0.2 \mathrm{~mm}$ in the control group $(\mathrm{p}<0.001)$ (Table 2$)$. The intra-observer ICC was 0.972 (95\% confidence interval [CI], 0.928-0.991) and the inter-observer ICC was 0.994 (95\% CI, 0.982-0.998).

The mean VAS score and Kujala score evaluated at the time of outpatient visit were 5.6 \pm 1.6 and $53.1 \pm 8.2$, respectively in the OS group. Regarding the correlation of the FPTP à and VAS and Kujala scores, the FPTP à showed a significantly negative correlation with VAS (-0.602) and a significantly positive correlation with the Kujala score $(0.708 ; \mathrm{p}<0.01$, both). Regarding the correlation of the TAP à and the VAS score and Kujala score, the TAP à revealed a significantly positive correlation with the VAS score (0.597) and a significantly negative correlation with the Kujala score $(-0.733 ; \mathrm{p}<0.01$, both). In the OS group, $43 \%$ showed substantial patellar tendinopathy or bone marrow edema at the tibial attachment site on T2-weighted sagittal images (Table 2 and Fig. 2). 
Table 2. Comparison of MRI Findings between the OS Group and the Control Group

\begin{tabular}{lccr}
\hline \multicolumn{1}{c}{ Characteristic } & $\begin{array}{c}\text { OS group } \\
(\mathrm{n}=30)\end{array}$ & $\begin{array}{c}\text { Control group } \\
(\mathrm{n}=30)\end{array}$ & p-value \\
\hline Insall-Salvati ratio & $0.97 \pm 0.2$ & $0.96 \pm 0.1$ & 0.817 \\
FPTP à, $(1) /(2)$ & $0.55 \pm 0.09$ & $0.77 \pm 0.11$ & $<0.001$ \\
TAP à, $(3) /(4)$ & $0.67 \pm 0.08$ & $0.29 \pm 0.07$ & $<0.001$ \\
Thickness of PT on tibial & $7.2 \pm 0.7$ & $4.0 \pm 0.2$ & $<0.001$ \\
attachment $(\mathrm{mm})$ & & & \\
Findings & & & \\
None & $3(10)$ & & \\
Peritendinous edema & $14(47)$ & & \\
Tendinopathy & $7(23)$ & & \\
Bone edema & $6(20)$ & & \\
Partial tear & 0 & & \\
\hline
\end{tabular}

Values are presented as mean \pm standard deviation or number (\%).

MRI: magnetic resonance imaging, OS: Osgood-Schlatter disease, FPTP: free patellar tendon proportion, TAP: tibial attachment proportion, PT: patellar tendon.

\section{Discussion}

This study revealed that the patellar tendon in the OS group was attached more widely to the tibial tuberosity, resulting in a reduced free tendon portion, and more proximally to the tibial articular surface compared with the control group. In addition, a positive correlation was found between the free portion of the patellar tendon and the clinical scores.

There has been some theoretical evidence that supports the results of the current study. If the patellar tendon is widely attached in the region of the tibial tuberosity, the potential increase of the stress acting on the ossicle can be inferred in case of OSD where the ossicle exists ${ }^{13,17,18}$. Furthermore, reduced portion of the free tendon also causes bigger stress on the insertion site due to shortening of the lever arm working on the tendon ${ }^{13,17,18)}$. More proximal attachment of the patellar tendon as was observed in the cases of OSD causes anterior translocation of the rotation axis in the knee joint, which thereby shortens the distance between the patellar tendon and the center of the rotation axis, and increases the stress on the attachment site of the patellar tendon in consequence ${ }^{9)}$. It has been also known to be related to the pain of the anterior knee joint experienced while performing non-extreme activities including squatting due to mobility and increased stress in cases where it is not ossified in the tibial tuberosity but rather remains as an ossicle. In these regards, the analysis on correlation of the FPTP à with the VAS score and Kujala score revealed a

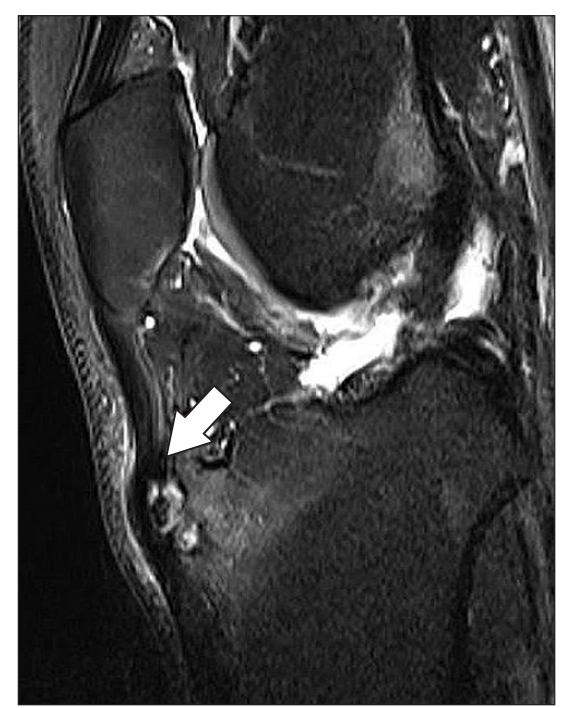

Fig. 2. T2-weighted magnetic resonance imaging scan. The white arrow indicates the thickened patellar tendon and bone marrow edema that contains the area of high signal intensity with the nonunited fragment.

significantly negative correlation with the VAS score and a significantly positive correlation with the Kujala score in the current study.

Some researches have provided the rationale for conducting ossicle resection ${ }^{4,19)}$. Furthermore, in cases where a continuous and strong extension mechanism is applied onto the separated ossicle through the patellar tendon, the ossicle separated from the tibial tuberosity presents a chronic mobility ${ }^{2,9,15,20)}$. Pihlajamaki et al. ${ }^{4)}$ conducted excision of the ossicle in 107 military personnel with OSD and observed the long-term progress. Their results showed that $87 \%$ returned to symptom-free normal daily life and $75 \%$ were able to return to the same sports activities they had enjoyed before surgery without symptoms. Nierenberg et al. ${ }^{21)}$ removed the ossicle with mobility in 22 patients beyond the completion of growth and reported that all patients returned to normal daily life symptom-freely within 3 months after surgery; emphasizing the importance of conducting preoperative evaluation on the presence of the ossicles that are separated and mobile. In addition, extension of the relatively shortened patellar tendon has been thought to be performed if the surrounding soft tissues are sufficiently dissected during ossicle excision with tibial tubercleplasty $^{4,13,22)}$.

About half (43\%) of the OS group in this study showed patellar tendinopathy and bone marrow edema. Although it is still controversial whether the symptoms of OSD are associated with the ossicle or patellar tendinopathy, patellar tendinopathy resulting from secondary degeneration of the patellar tendon caused after 
separation from the tibial tuberosity is one of the characteristics of the disease ${ }^{9,15,23)}$. Hirano et al. ${ }^{9)}$ conducted analysis on MRI findings and reported that $69 \%$ of the patients who were in the progress stage and all patients who were in the late stage showed the condition of the patellar tendinitis including the increase in the thickness of the attachment site of the patellar tendon. They predicted that there would be inflammatory reaction surrounding the secondary ossification with progression of OSD and the relaxation of the symptoms would be related to the recovery of the patellar tendinitis. We think that the symptom was continuously present even after the ossicle was excised either with an open procedure or an arthroscopic procedure because the patellar tendinopathy associated with the attachment site of the patellar tendon was not treated appropriately; therefore, an MRI evaluation not only for the osseous tissues but also for the soft tissues should be done appropriately prior to surgery ${ }^{9,13)}$.

The limitations of this study are as follow. First, the nature of our institution allowed the study to be based on male patients only and caused a male-weighted trend in terms of the gender ratio. Second, this study did not conduct a comparison with asymptomatic patients with the ossicle. Third, the study was limited to the ossicle type OSD excluding other types of OSD. A prospective study that determines the relationship between preoperative MRI and symptoms and the correlation between postoperative MRI and relaxation of symptoms is required. In addition, the results caused by the pressure loaded onto the attachment site of the patellar tendon based on the modality of the patellar tendon attachment should be supported by biomechanical studies. Despite numerous limitations, this study has significance in terms of the rarity of the study on this subject both domestically and internationally, inclusion of the relatively larger number of patients in the experimental group and control group, and the achievement of the results among young adults beyond the growth period. In addition, there was no intergroup difference in the location of the patella that was evaluated using the Insall-Salvati ratio and measurements for calculation of the ratio were based on the inferior region of the patella and the tibial articular surface to rule out the influence of individual variations such as patellar alta and baja. The authors believe that the MRI characteristic of OSD described this study can be useful for predicting the prognosis of the disease and planning proper treatment methods for young adult patients beyond the growth period with symptomatic OSD, especially athletes and military personnel engaged in activities that increase the stress on the knee joint.

\section{Conclusions}

In young adults with OSD, the MRI revealed a relatively less free portion and relatively more proximal attachment of the patellar tendon compared to those of young adults without OSD. Furthermore, positive correlations were found between the free portion of the patellar tendon and the clinical scores in the OSD patients. In future research, detailed analysis on the patellar tendon attachment should be conducted since secondary patellar tendinopathy is also frequently encountered in patients with OSD.

\section{Conflict of Interest}

No potential conflict of interest relevant to this article was reported.

\section{References}

1. Cohen B, Wilkinson RW. The Osgood-Schlatter lesion; a radiological and histological study. Am J Surg. 1958;95:731-42.

2. Gholve PA, Scher DM, Khakharia S, Widmann RF, Green DW. Osgood Schlatter syndrome. Curr Opin Pediatr. 2007; 19:44-50.

3. Eun SS, Lee SA, Kumar R, Sul EJ, Lee SH, Ahn JH, Chang MJ. Direct bursoscopic ossicle resection in young and active patients with unresolved Osgood-Schlatter disease. Arthroscopy. 2015;31:416-21.

4. Pihlajamaki HK, Mattila VM, Parviainen M, Kiuru MJ, Visuri TI. Long-term outcome after surgical treatment of unresolved Osgood-Schlatter disease in young men. J Bone Joint Surg Am. 2009;91:2350-8.

5. Weiss JM, Jordan SS, Andersen JS, Lee BM, Kocher M. Surgical treatment of unresolved Osgood-Schlatter disease: ossicle resection with tibial tubercleplasty. J Pediatr Orthop. 2007;27:844-7.

6. Narayan N, Mitchell PD, Latimer MD. Complete resolution of the symptoms of refractory Osgood-Schlatter disease following percutaneous fixation of the tibial tuberosity. BMJ Case Rep. 2015 Feb 12 [Epub]. http://dx.doi.org/10.1136/ bcr-2014-206734.

7. Beyzadeoglu T, Inan M, Bekler H, Altintas F. Arthroscopic excision of an ununited ossicle due to Osgood-Schlatter disease. Arthroscopy. 2008;24:1081-3.

8. Topol GA, Podesta LA, Reeves KD, Raya MF, Fullerton BD, Yeh HW. Hyperosmolar dextrose injection for recalcitrant 
Osgood-Schlatter disease. Pediatrics. 2011;128:e1121-8.

9. Hirano A, Fukubayashi T, Ishii T, Ochiai N. Magnetic resonance imaging of Osgood-Schlatter disease: the course of the disease. Skeletal Radiol. 2002;31:334-42.

10. El-Husseini TF, Abdelgawad AA. Results of surgical treatment of unresolved Osgood-Schlatter disease in adults. J Knee Surg. 2010;23:103-7.

11. Orava S, Malinen L, Karpakka J, Kvist M, Leppilahti J, Rantanen J, Kujala UM. Results of surgical treatment of unresolved Osgood-Schlatter lesion. Ann Chir Gynaecol. 2000; 89:298-302.

12. Cakmak S, Tekin L, Akarsu S. Long-term outcome of Osgood-Schlatter disease: not always favorable. Rheumatol Int. 2014;34:135-6.

13. Demirag B, Ozturk C, Yazici Z, Sarisozen B. The pathophysiology of Osgood-Schlatter disease: a magnetic resonance investigation. J Pediatr Orthop B. 2004;13:379-82.

14. Kadakia NR, Ilahi OA. Interobserver variability of the InsallSalvati ratio. Orthopedics. 2003;26:321-3.

15. Rosenberg ZS, Kawelblum M, Cheung YY, Beltran J, Lehman WB, Grant AD. Osgood-Schlatter lesion: fracture or tendinitis? Scintigraphic, CT, and MR imaging features. Radiology. 1992;185:853-8.

16. Zissen MH, Wallace G, Stevens KJ, Fredericson M, Beaulieu CF. High hamstring tendinopathy: MRI and ultrasound imaging and therapeutic efficacy of percutaneous corticosteroid injection. AJR Am J Roentgenol. 2010;195:993-8.
17. Karlsson J, Bunketorp O, Lansinger O, Romanus B, Sward L. Lowering of the patella secondary to anterior advancement of the tibial tubercle for the patellofemoral pain syndrome. Arch Orthop Trauma Surg. 1986;105:40-5.

18. Tsaopoulos DE, Baltzopoulos V, Maganaris CN. Human patellar tendon moment arm length: measurement considerations and clinical implications for joint loading assessment. Clin Biomech (Bristol, Avon). 2006;21:657-67.

19. Flowers MJ, Bhadreshwar DR. Tibial tuberosity excision for symptomatic Osgood-Schlatter disease. J Pediatr Orthop. 1995;15:292-7.

20. Nakase J, Aiba T, Goshima K, Takahashi R, Toratani T, Kosaka M, Ohashi Y, Tsuchiya H. Relationship between the skeletal maturation of the distal attachment of the patellar tendon and physical features in preadolescent male football players. Knee Surg Sports Traumatol Arthrosc. 2014;22:195-9.

21. Nierenberg G, Falah M, Keren Y, Eidelman M. Surgical treatment of residual osgood-schlatter disease in young adults: role of the mobile osseous fragment. Orthopedics. 2011;34:176.

22. DeBerardino TM, Branstetter JG, Owens BD. Arthroscopic treatment of unresolved Osgood-Schlatter lesions. Arthroscopy. 2007;23:1127.

23. Kaya DO, Toprak U, Baltaci G, Yosmaoglu B, Ozer H. Longterm functional and sonographic outcomes in OsgoodSchlatter disease. Knee Surg Sports Traumatol Arthrosc. 2013;21:1131-9. 